\title{
Epidemiology and Clinical Description of Amyotrophic Lateral Sclerosis in Low Income Setting: A Syndrome with Short Survival
}

\author{
Komi Assogba ${ }^{1, ~}$, Souleymane Brah ${ }^{2}$, Damelan Kombate ${ }^{1}$, Kossivi M. Apetse ${ }^{1}$, Rabi Barry-Barque ${ }^{1}$, \\ Mofou Belo ${ }^{3}$, Koffi A. A. Balogou ${ }^{1}$ \\ ${ }^{1}$ Neurology service, Campus University Teaching Hospital, Lome, Togo \\ ${ }^{2}$ Department of Internal medicine, Niamey University Teaching Hospital, Niamey, Niger \\ ${ }^{3}$ Neurological Clinic, Sylvanus Olympio University Hospital of Lome, Lome, Togo
}

\section{Email address:}

seraphinassogba@hotmail.com (K. Assogba)

\section{To cite this article:}

Komi Assogba, Souleymane Brah, Damelan Kombate, Kossivi M. Apetse, Rabi Barry-Barque, Mofou Belo, Koffi A. A. Balogou. Epidemiology and Clinical Description of Amyotrophic Lateral Sclerosis in Low Income Setting: A Syndrome with Short Survival. American Journal of Psychiatry and Neuroscience. Special Issue: Clinical Neurosciences in Tropical Practice. Vol. 3, No. 5-1, 2015, pp. 5-8. doi: 10.11648/j.ajpn.s.2015030501.12

\begin{abstract}
Background: Amyotrophic lateral sclerosis (ALS) is a degenerative motor neuron disease. It evolves to loss of autonomy and death. Objective: To describe the cases of ALS clinically definite observed in hospital field. Patients and methods: The retrospective study has covered a period of 10 years (2003-2012) and a total of 10,128 patient's files were analyzed. The neurology department of our level 3 hospital has located the current study. Six patients with no particular medical history have been reported. The inclusion patients are cases where the diagnosis of ALS was clinically definite according to the modified El Escorial classification. About 978 of likely, possible and probable cases of ALS were not included. Results: The mean age was 49 years (24 and 67), all males, with a frequency of $0.59 \%$ and an incidence rate of 0.6 cases per year. The clinical signs were marked by the pyramidal syndrome and peripheral neuropathic motor syndrome. MRI or CT scan signs were marked by the bulbar light atrophy. EMG had shown spontaneous activities with reinnervation signs. The histology exam has found neurogenic fascicular atrophy. The average duration of progression of the disease from the diagnostic to death was 17.6 months (6 to 36). The median of survival all cases combined was 42 weeks. Symptomatic treatment was associated to rehabilitation. Riluzole has been established despite its high cost, but without success. Conclusion: This observation highlighted the major difficulties encountered in the management of ALS and its increasing frequency in south Saharan Africa.
\end{abstract}

Keywords: Amyotrophic Lateral Sclerosis, Degenerative Diseases, Motor Neuron

\section{Introduction}

Amyotrophic lateral sclerosis (ALS) or Lou Gehrig's disease is a neurodegenerative disease of both motor neuron pathways, leading to loss of independence and death in mean term. It is revealed by progressive motor deficits that affecting members and oropharyngeal muscles. ${ }^{[1-3]}$ There is no curative cure up today and the one available, riluzole, only slow the disease progression in case of early diagnostic. The treatment is then symptomatic with a multidisciplinary team. ${ }^{[1,4]}$ The incidence is 1 in 100,000 peoples per year with a peak age between 55 and 70 years old and the prevalence is 0.45 to $0.89 / 100,000$ with a male predominance. ${ }^{[5,6]}$

\section{Subjects and Methods}

It was a retrospective and descriptive study covered a period of 10 years (2003-2012). A total of 10,128 patient's files were analyzed. The neurology department of our tertiary hospital had located the present study. The included patients were those who met the criteria of clinically definite ALS according to the consensus of revised El Escorial classification. ${ }^{[7]}$ All blood and CSF routine analysis were normal. Brain imaging was performed for exclusion diagnostic. Electromyography (EMG) recording and neuromuscular biopsy with histology analysis were the associated tools to retain the definite diagnostic of ALS. 
Genetics studies were not available. Many unfulfilled files and the likely, probable and possible cases of ALS were rejected. The patients or relatives have given their inform consent. The study was approved by the ethic committee of the university hospital.

\section{Results}

Six patients were retained according to the above criteria. All patients were those with ALS clinically definite. The frequency of ALS was $0.59 \%$ and the incidence was 0.6 case /year. The average age was 49 years with extremes of 24 and 67. All patients were males. The median age of diagnosis was 51 years and mean time to death after the disease onset was 30 months. The clinical signs were marked by the pyramidal syndrome and peripheral neuropathic motor syndrome. MRI or CT scan signs were normal in 3 cases and marked by the bulbar and upper cervical medulla atrophy in other cases. EMG had shown spontaneous activities with reinnervation signs. The histological exam had found neurogenic fascicular atrophy. The average duration of progression of the disease from the diagnostic to death was 17.6 months (6 to 36). The median of survival all cases combined was 42 weeks. The summary of clinical and sociodemographic data of the six patients is reported in table I.

Table I. Summary of clinical and demographic features of the six patients.

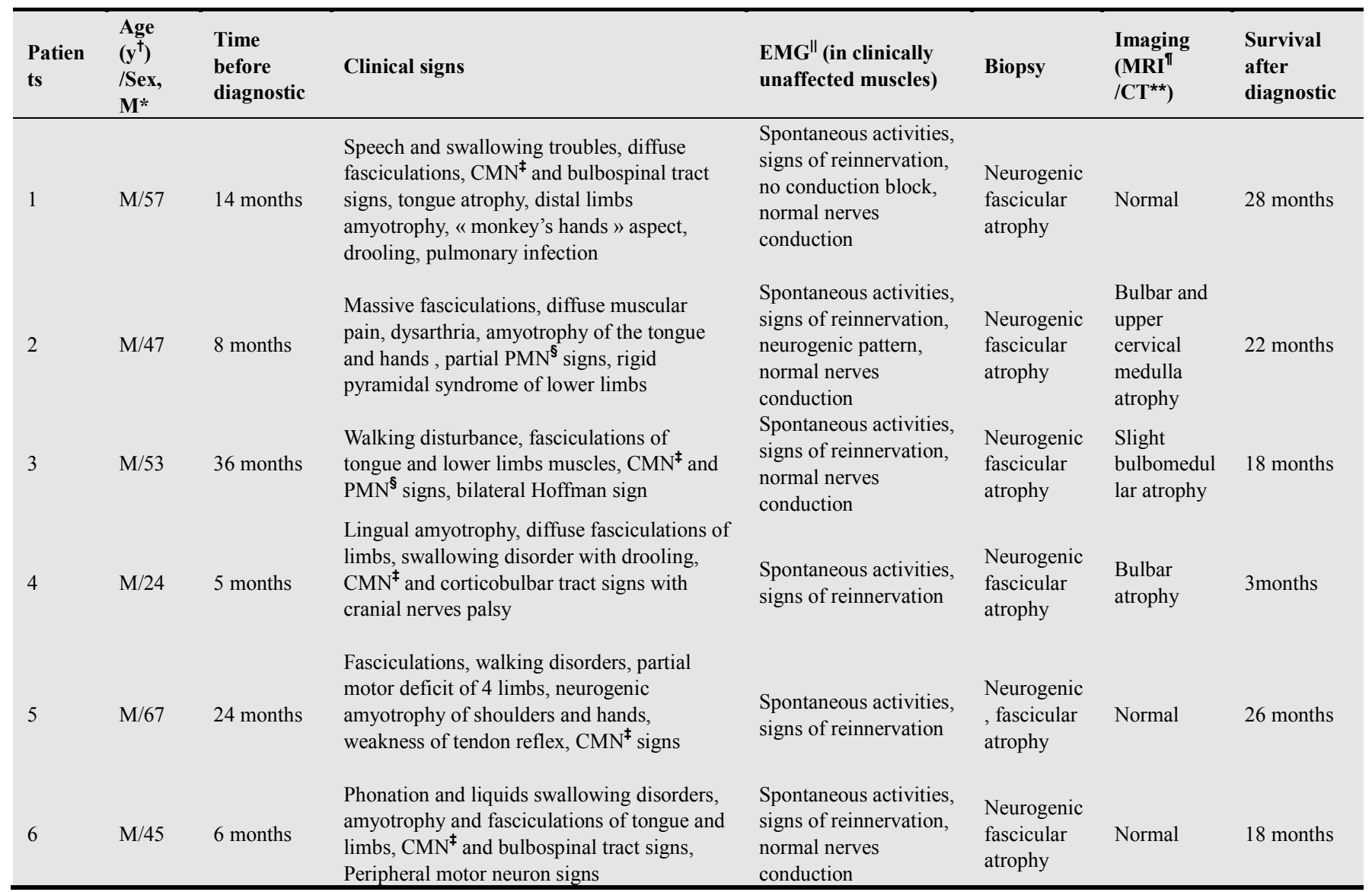

Legend: $\mathrm{M}^{*}=$ male; $\mathrm{y}^{\dagger}=$ years; $\mathrm{CMN}^{\ddagger}=$ Central motor neuron signs; $\mathrm{PMN}^{\S}=$ Peripheral motor neuron signs; EMG $^{\|}$:electromyography; MRI $^{\Uparrow}$ : Magnetic Resonance Imaging; $\mathrm{CT}^{\star *}=$ computer tomography

\section{Discussion}

\subsection{Methodology}

We have reported 6 cases of ALS clinically definite in a period of 10 years. This retrospective clinical cases study has some weaknesses. It concerns solely the patients who were followed up in neurology. The diagnosis is based on clinical signs and symptoms of ALS. The patients with likely and possible diagnostic of ALS were rejected. In other hand, some ALS clinically definite may not expect the hospitals, and used to attend basic clinics. All these recruitment bias reduced the sample size and show that the study is not exhaustive. However, the consistency and relevance of our results with the literature give validity to the reported data.

\subsection{Epidemiological Aspects}

The age of $1^{\text {st }}, 3^{\text {rd }}, 5^{\text {th }}$ and $6^{\text {th }}$ patients is similar to that reported in the literature, but the $2^{\text {nd }}$ and $4^{\text {th }}$ are younger than the peak age of onset reported in several previous studies. ${ }^{[1,5,6]}$

The juvenile forms may have a familial origin in $30 \%{ }^{[8-10]}$ The presence of juvenile forms should lead to perform genetic testing in search of SOD1 mutation in a young patient. 
In our case, a family survey was conducted in patient age of 24 years, in the lack of the genetic studies.

The reported prevalence in this study of ALS may be biased by undiagnosed. Indeed, cases of probable or possible ALS which should be confirmed by appropriated diagnostic tools were not included. The suspected patients must pay themselves for the clinical investigations. EMG and histology are not locally available and the patient must travel to abroad country to perform these tests. Data from different hospitals scattered studies in Africa show that ALS is a reality in the continent, but still rare. ${ }^{[1,3,5]}$

\subsection{Clinical Features of ALS}

Concerning our patients, the presence of loss of 2 spinal territories of the peripheral and one territory of central motor neurons at least is compatible with the El Escorial criteria for clinically definite ALS. To these signs, are added the gradual evolution and especially the absence of sensory, visual, sphincters, autonomic and basal ganglia disturbances. ${ }^{[7]}$ The diagnosis of ALS is based on clinical criteria of El Escorial revised in 1998, but some additional tests (EMG and neuromuscular histology) can confirm the diagnosis or eliminate certain diseases that may mimic it. There are diseases that mimic ALS where the affected nerve territory is either only at the central or peripheral motor neurons..$^{[11-14]}$ These diseases have been eliminated through careful neurological examination and biological and radiological investigations. ALS is a progressive and irreversible disease.

Brain imaging (MRI and CT) is not the tool to confirm or to reject the ALS diagnosis, as it can be normal in many cases in where it was done. These observations raised the capital interest of a thorough neurological examination as the solely diagnostic weapon we have in African regions to diagnosis ALS.

Regarding the course of the disease, we believe that there must been a delay in diagnosis of patients 3 and 5, because they have generalized their symptoms, with all the different clinical phases of ALS. The onset time of symptoms was 2 years for the $3^{\text {rd }}$ and 3 years for the $5^{\text {th }}$. For other patients, it may be a case for rapid evolution or the onset of the disease has been poorly evaluated. Diagnostic delays affect about $25 \%$ of patients who died before they meet the criteria of the disease. ${ }^{[15,16]}$ These diagnostics are mostly encountered delays in cases where the clinic is rough, often misleading and mimic many other neurological disorders.

The existence of cognitive impairment should not exclude the diagnosis of ALS. Indeed signs of fronto temporal lobe dementia (FTD) may occur before or after the ALS symptoms in $40 \%$ of cases especially in the bulbar form. ${ }^{[17]}$

\subsection{ALS Management}

Concerning the medical care, only symptomatic treatment was offered to our patient. Treatment with Riluzole has been established for those who were able to buy it on abroad because it is non- availability in the country. In these cases where Riluzole was used, any significant improvement was not observed. In the literature and according to different consensus, typical ALS support is based on three lines:

1-Neuroprotective treatment made by glutamate-release antagonists (Riluzole, Verapamil, Topiramate) which has been shown to slow the course of ALS, but this does not proved its effective effects in several studies. ${ }^{[2,3,18,19]}$

2-Symptomatic treatment should be done by multidisciplinary team to improve the quality of life, ${ }^{[1]}$ and finally followed by palliative and end-of-life cares. ${ }^{[2]}$

Noninvasive positive pressure ventilation (NIPPV) is a therapeutic option used for many patients with less than $50 \%$ of vital capacity. The NIPPV may be replaced by a tracheostomy with permanent ventilation if necessary. The use of mechanical ventilation varies between countries with cross-cultural and ethical differences. Many studies suggest that the use of NIPPV improves survival and quality of life in patients admitted to multidisciplinary team centers. ${ }^{[20,21]}$

The introduction of percutaneous endoscopic gastrostomy (PEG) has given a real comfort to patients suffering from severe swallowing disorders. ${ }^{[22]}$ The drooling, bronchial secretions, insomnia and pseudobulbar signs were supported by anti emetics, amitriptyline, atropine, and physiotherapy. ${ }^{[3,4,23]}$

The management of neuralgic pain was provided by good nursing and a delicate engagement with regular change of position of the patient. The adjuvant treatment includes analgesics, anti-inflammatory and antispasmodics. ${ }^{[1,2,4,23,24]}$

The patient quality of life depends strongly on medical, psychosocial and existential accompany in life. Fatigue, depression, anxiety and hopelessness are common among these patients. The treatment of these symptoms improves significantly the quality of life of patient with ALS. ${ }^{[23-25]}$

\section{Conclusion}

Carefully clinical examination is essential in case of suspected ALS. The management of ALS remains difficult in all continents. The ALS is still under diagnosis in Africa. The data on this disease are highly variable, rare and sparse in Africa.

\section{References}

[1] Van den Berg JP, Kalmijn S, Lindeman E, Veldink JH, de Visser M, Van der Graaff MM, et al. Multidisciplinary ALS care improves quality of life in patients with ALS. Neurology 2005;65:1264-67.

[2] Mitsumoto H, Rabkin JG. Palliative care for patients with amyotrophic lateral sclerosis: "prepare for the worst and hope for the best". JAMA 2007;298:207-16.

[3] Bensimon G, Lacomblez L, Delumeau JC, Bejuit R, Truffinet $\mathrm{P}$, Meininger $\mathrm{V}$, et al. A study of riluzole in the treatment of advanced stage or elderly patients with amyotrophic lateral sclerosis. J Neurol 2002;249(5):609-15.

[4] Practice advisory on the treatment of amyotrophic lateral sclerosis with riluzole: report of the Quality Standards 
Subcommittee of the American Academy of Neurology. Neurology 1997;49:657-62.

[5] Rowland LP. Amyotrophic lateral sclerosis. Curr Opin Neurol Neurosurg 1994;7:310-15.

[6] Mitchell JD, Borasio GD. Amyotrophic lateral sclerosis. Lancet 2007;369:2031-41.

[7] Brooks BR, Miller RG, Swash M, Munsat TL, et al. World Federation of Neurology Research Group on Motor Neuron Diseases. El Escorial revisited: revised criteria for the diagnosis of amyotrophic lateral sclerosis. Amyotroph Lateral Scler Other Motor Neuron Disord 2000;1:293-99.

[8] Wiedau-Pazos M, Goto JJ, Rabizadeh S, Gralla EB, Roe JA, Lee MK. Altered reactivity of SOD1 in FALS. Science 1996; 271:515-18

[9] Allen S, Heath PR, Kirby J, Wharton SB, Cookson MR, Menzies FM, et al. Analysis of the cytosolic proteome in a cell culture model of FALS reveals alterations to the proteasome, antioxidant defenses and nitric oxide synthetic pathways. J Biol Chem 2003;278:6371-83.

[10] Hand CK, Khoris J, Salachas F, Gros-Louis F, Lopes AA, Mayeux-Portas V, et al. A novel locus for familial amyotrophic lateral sclerosis on chromosome 18q. Am J Hum Genet 2002;70:251-56.

[11] Delmont E, Roth S, Heudier P, Cua E, Kaphan R, Campagni JP, et al. Primary hyperparathyroidism, a differential diagnosis of motor neuron diseases. Rev Med Interne 2001;22:1253-55.

[12] Traynor BJ, Codd MB, Corr B, Forde C, Frost E, Hardiman OM. Amyotrophic lateral sclerosis mimic syndromes: a population-based study. Arch Neurol 2000;57:1171-76.

[13] Eymard B. Polymyositis, dermatomyositis and inclusion body myositis, nosological aspects. Presse Med 2003;32:1656-67.

[14] Attout H, Rahmeh F, Ziegler F. Gougerot-Sjögren syndrome simulating amyotrophic lateral sclerosis. Rev Med Interne 2000;21:708-12.

[15] Verma A, Berger JR, Snodgrass S, Petito C. Motor neuron disease: a paraneoplastic process associated with anti-hu antibody and small-cell lung carcinoma. Ann Neurol 1996;
40:112-6.

[16] Le Forestier N, Maisonobe T, Piquard A, Rivaud S, CrevierBuchman L. Does primary lateral sclerosis exist? A study of 20 patients and a review of the literature. Brain 2001;124:1989-99.

[17] Lomen-Hoerth C. Characterization of amyotrophic lateral sclerosis and frontotemporal dementia. Dement Geriatr Cogn Disord 2004;17:337-41.

[18] Sorenso EJ, Windbank AJ, Mandrekar JN, Bamlet SH. Subcutaneous IGF-1 is not beneficial in 2-year ALS trial. Neurology 2008;71:1770-75.

[19] Gordon PH, Moore DH, Miller RG, Florence JM, Verheijde JL, Doorish C, et al. Efficacy of minocycline in patients with amyotrophic lateral sclerosis: a phase III randomised trial. Lancet Neurol 2007;6:1045-53.

[20] Bourke SC, Tomlinson M, Williams TL, Bullock RE, Shaw PJ, Gibson GJ. Effects of non-invasive ventilation on survival and quality of life in patients with amyotrophic lateral sclerosis: a randomised controlled trial. Lancet Neurol 2006;5:140-47.

[21] Rabinstein AA, Wijdicks EF. Warning signs of imminent respiratory failure in neurological patients. Semin Neurol 2003;23:97-102.

[22] Kasarskis EJ, Scarlata D, Hill R, Fuller C, Stambler N, Cedarbaum JM. A retrospective study of percutaneous endoscopic gastrostomy in ALS patients during BDNF and CNTF trials. J Neurol Sci 1999;169:118-25.

[23] McDermott CJ, Shaw PJ. Diagnosis and management of motor neuron diseases. BMJ 2008;336:658-62.

[24] Jackson CE, Gronseth G, Rosenfeld J, Barohn RJ, Dubinsky R, Simpson CB, et al. Randomized double-blind study of botulium toxin type B for sialorrhea in ALS patients. Muscle Nerve 2009;39:137-43.

[25] Rosen HJ, Cummings J. A real reason for patients with pseudobulbar affect to smile. Ann Neurol 2007; 61:92-6. 